\title{
Unmoderated Posters: Trauma, Reconstruction and Diversion
}

\begin{abstract}
UP-56
The Effect of Preoperative Urine Culture Status on Urethroplasty Complications and Outcomes

Rourke, Keith; Lavoie, Callum; Kinnaird, Adam

University of Alberta, Edmonton, $\mathrm{AB}$, Canada

Objectives: Urethroplasty remains the most definitive treatment for urethral stricture. Many patients with urethral stricture experience urinary tract infection or have chronic bacteruria. It is unclear if colonization of the urinary tract affects urethroplasty outcomes. The objective of this study is to evaluate the effect of a positive preoperative urine culture on urethroplasty complications and outcomes.

Methods: A retrospective review of 734 urethroplasties performed by a single surgeon was performed. All patients received preoperative culture sensitive parenteral antibiotics. Positive urine culture was defined as $>108$ bacteria per $\mathrm{ml}$ or in select instances $>106$ of specific pathogenic bacteria. The effect of a positive urine culture was investigated by examining differences in postoperative wound infection, UTI, and stricture recurrence. Statistical analysis was performed using a Fisher's 2-sided exact test.

Results: Overall the rates of wound infection, UTI and stricture recurrence were $4.7 \%, 4.0 \%$ and $8.7 \%$ respectively. Comparison between the positive urine culture group and the negative urine culture group revealed a statistically significant difference in the average age of the patients $(p=0.0001)$, but otherwise no statistically significant differences in average stricture length $(p=0.9781)$, revision urethroplasty $(p=0.05231)$ or average co-morbidity score $(p=0.1086)$. Between the positive and negative urine culture groups, the rate of postoperative wound infection were not statistically significant $(p=0.5587)$. Likewise, the rates of postoperative UTI $(p=1.000)$ and stricture recurrence $(p=0.2965)$ were not found to be significantly different with a mean follow-up of 50 months. The specific type of pathogen did not alter the rate of complications.

Conclusions: The presence of a positive urine culture with administration of clinically appropriate antimicrobials does not appear to affect urethroplasty complications or outcomes.
\end{abstract}

\section{UP-57}

Urologic Morbidity and Access to Care among Traumatic Spinal Cord Injured Patients

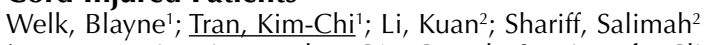

${ }^{1}$ Western University, London, ON, Canada; ${ }^{2}$ Institute for Clinical Evaluative Sciences, London, ON, Canada

Purpose: To assess access to urologic care among traumatic $\mathrm{SCl}$ patients in Ontario.

Methods: Administrative data records from the Ontario Health Insurance Plan, Discharge Abstract Databases, and the National Rehabilitation System were linked. The primary outcome was urologic consultation. The primary exposure was the year of injury. Measured covariates included lesion level, age, gender, comorbidity burden, and socioeconomic status. Logistic regression models were used to assess for linear trends over time. A Cox proportional hazards model was used to assess the impact of covariates on urologic consultation among TSCI patients.

Results: We identified 1551 incident TSCI patients who were discharged from a rehabilitation hospital in Ontario between 2002-2012. The mean followup time of this cohort was 5.3 (SD 2.7) years. Within this cohort, $74 \%$ were male, and the mean age was 48 (SD 19) years. $69 \%$ of patients $(1065 / 1551)$ were seen by a urologist a mean of 2 (SD 2.5) years after SCl.
Urologic referral was generally initiated by family practitioners $(37 \%)$, and physiatry $(27 \%)$. Over the study period there was no significant linear trend in the proportion of TSCl patients being assessed by a urologist within 1 year (mean 55\%, $\mathrm{p}=0.97$ ) or 2 years (mean $62 \%, \mathrm{p}=0.75$ ) of their initial injury. A Cox proportion hazards model demonstrated that TSCI patients who were female (HR $0.78,95 \% \mathrm{Cl} 0.66-0.92)$ or over 65 years of age (HR $0.70,95 \% \mathrm{Cl} 0.57-0.85$ ) were significantly less likely to be referred to a urologist. Year of injury, level of TSCl, socioeconomic status, and comorbidity level were not significant predictors.

Conclusions: Urologists are often not involved in the post rehabilitation management of TSCl patients, and this has not changed significantly over the last 10 years. Females, and older patients, are significantly less likely to be referred to a urologist. Further research into the impact of these practice patterns on patient morbidity is needed.

\section{UP-58}

Penile Amputation and Successful Reattachment: Role of Winter Shunt in Postoperative Viability: A Case Report and Review of Literature

Fuoco, Michael ${ }^{1}$; Cox, Leonard ${ }^{2}$; Kinahan, Thomas ${ }^{3}$

${ }^{1}$ Queen's University, Department of Urology, Kingston, ON, Canada; ${ }^{2}$ Victoria General Hospital, Victoria, BC, Canada; ${ }^{3}$ University of British Columbia; Kelowna General Hospital, Kelowna, BC, Canada

Introduction and Objectives: Amputation of the penis is a rare occurrence and reattachment surgery is difficult and may fail due to lengthy warm ischemic time, difficulties with tissue destruction, and vascular compromise. Based on the vascular physiology of the penis, there is an argument for performing a shunt between the cavernosum and the spongiosum at the time of reattachment surgery, to allow better blood flow to the amputated portion. Methods: A 53-year-old patient with paranoid schizophrenia presented 2 hours following penile amputation. He had swallowed the excised portion, which was endoscopically retrieved from the stomach in the emergency department. Successful reattachment was achieved including microvascular repair. A Winter shunt using a 14 gauge biopsy needle was performed to improve venous circulation.

Results: The patient has been followed for 3 years from the date of repair, and has adequate erection for intercourse, but has sensory loss over the dorsal aspect and glans. A series of 5 penile reimplantations following amputation is presented, with 3 of 5 successful. Only one of these has had a shunt procedure. Factors related to success and failure are individually characterized.

Conclusions: The role of spongiocavernosal shunting at the time of surgical replantation of an amputated penis is unclear due to the rarity of penile amputation, but may have played a role in the success of our index case. While other factors may play a greater role in success, it makes physiological sense to perform this shunt to improve the chances of success in all cases where possible. Because of the nature of the patients involved, long term follow-up is difficult to achieve. 